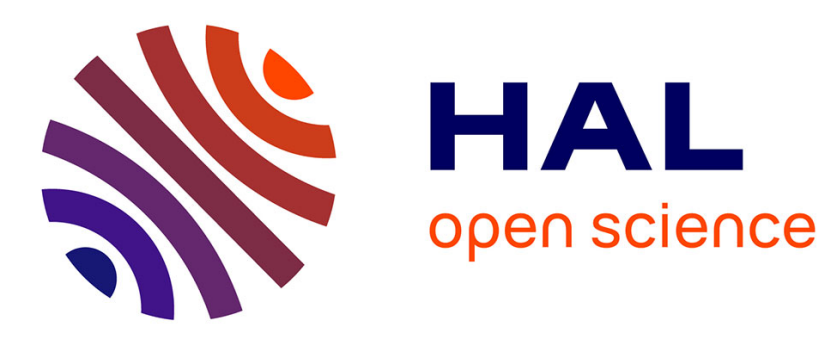

\title{
Indirectly coupled films
}

Jean-Claude Bruyere, G. Clerc, Olivier Massenet, D. Paccard, Robert Montmory, Louis Néel, J. Valin, A. Yelon

\section{To cite this version:}

Jean-Claude Bruyere, G. Clerc, Olivier Massenet, D. Paccard, Robert Montmory, et al.. Indirectly coupled films. IEEE Transactions on Magnetics, 1965, MAG1 (3), pp.174-180. 10.1109/TMAG.1965.1062946 . hal-02887452

\section{HAL Id: hal-02887452}

\section{https://hal.science/hal-02887452}

Submitted on 2 Jul 2020

HAL is a multi-disciplinary open access archive for the deposit and dissemination of scientific research documents, whether they are published or not. The documents may come from teaching and research institutions in France or abroad, or from public or private research centers.
L'archive ouverte pluridisciplinaire HAL, est destinée au dépôt et à la diffusion de documents scientifiques de niveau recherche, publiés ou non, émanant des établissements d'enseignement et de recherche français ou étrangers, des laboratoires publics ou privés. 


\section{REFERENCES}

[1] H. Suhl, "The nonlinear behavior of ferrites at high microwave signal levels," Proc. IRE, vol. 44, pp. 1270-1284, October 1956

[2] H. Suhl, "The theory of ferromagnetic resonance at high signal powers," J. Phys. Chem. Solids (GB), vol. 1, pp. 209-227, 1957.

[3] For a recent review, see R. Damon, Magnetism, vol. 1, G. Rado and H. Suhl (Eds.). New York, London: Academic, 1963.

[4] J. J. Green and E. Schlömann, "High power ferromagnetic resonance at $X$-band in polycrystalline garnets and ferrites," IRE Trans. on Microwave Theory and Techniques, vol. MTT-8, pp. 100-103, January 1960 .

[5] E. Schlömann, J. J. Green, and U. Milano, "Recent developments in ferromagnetic rasonance at high power levels," $J$. Appl. Phys., vol. 31, pp. 386S-395S, May 1960.

[6] J. J. Green, J. H. Saunders, and E. Schlömann, "Ferrite materials for microwave applications at high peak-power levels," Prac. IEE (London), vol. 109, pt. B, pp. 75-80, June 1961.

[7] H. Suhl, "Ferrite microwave devices for use at high signal energy levels,' U.S. Patent 2883629.

[8] W. W. Malinowsky and R. W. Babbitt, "Fine-grained ferrites. III. $\mathrm{Ni}_{1}-\mathrm{Co}_{x} \mathrm{Fe}_{2} \mathrm{O}_{4}$. High-power microwave and $\mathrm{RF}$ properties," J. Appl. Phys., vol. 35, pp. 1012-1014, March 1964.
[9] J. J. Green, J. S. Waugh, and B. J. Healy, "Microwave properties of fine-grain nickel ferrite," J. Appl. Phys., vol. 35, pp. 1006-1007, Warch 1964.

[10] B. Lax and K. J. Button, Microwave Ferrites and Ferrimagnetics. New York: McGraw-Hill, 1962, pp. 323-354.

[11] B. Lax, K. J. Button, and L. M. Roth, "Ferrite phase shifters in rectangular waveguide," $J$. A ppl. Phys., vol. 25, pp. 14131421, November 1954.

[12] W. Yon Aulock, Ferrite Devices for Microwave Application. Englewood Cliffs, N. J.: Prentice-Hall, to be published.

[13] M. A. Treuhaft and L. M. Silber, "Use of microwave ferrite toroids to eliminate external magnets and reduce switching power," Proc. IRE (Correspondence), vol. 46, p. 1538, August 1958.

[14] E. Schlömann, "Theoretical analysis of twin slab phase shifters in rectangular waveguide," presented at the 1965 G-MTT Symp., Clearwater, Fla.

[15] E. Schlömann, "Spin-wave analysis of ferromagnetic resonance in polycrystalline ferrites," $J$. Phys. Chem. Solids (GB), vol. 6 , no. $2 / 3$, pp. $242-256,1968$.

[16] E. A. Maguire and J. J. Green, "Ferrite materials with temperature stabilized magnetizations, II: Gadolinium-yttrium-ironindium garnets," J. Am. Ceram. Soc., to be published.

\title{
Indirectly Coupled Films
}

\author{
J. C. BRUYÈRE, G. ClERC, O. MASSENET, D. PACCARD, R. MONTMORY, \\ L. NEEL, J. VALIN, AND A. YELON, MEMBER, IEEE
}

\begin{abstract}
A positive coupling has been observed between magnetic films separated by a thin metallic layer. Five methods which have been developed for measuring this coupling and the variation of the coupling with evaporation temperature, measuring temperature, and thickness of the intermediate layer are described. Three mechanisms are proposed for the observed coupling: bulk diffusion of ferromagnetic atoms into the intermediate layer, diffusion of magnetic atoms along grain boundaries, and polarization of conduction electrons. The effect of this coupling on slow switching, pulse switching, and creep is discussed.
\end{abstract}

\section{INTRODUCTION}

$\mathbf{A}^{\mathrm{T}}$ THE BEGINNING of this century, Maurain [1], [2] performed a series of experiments in which he electroplated copper and then iron onto a magnetized iron wire and observed that the electroplated iron became magnetized in the same direction as the wire, provided the copper was not too thick. He was unable at the time to explain these results, and they have remained unexplained since. This has led us to attempt to reproduce his study in a somewhat different form, which we consider is more readily interpretable.

We have prepared, by vacuum evaporation, a series of

Manuscript received March 25, 1965. The work reported in this paper was supported by C.N.R.S. and D.G.R.S.T. under Contract 62 FR 163. This paper was presented at the 1965 INTERMAG Conference, Washington, 1). C.

J. C. Bruyère, G. Clerc, O. Massenet, D. Paccard, R. Montmory, L. Néel, and A. Yelon are with the Laboratoire d'Electrostatique et de Physique du Metal, Grenoble, France.

J. Valin is with the Societe d'Electronique et d'Automatisme, Courbevoie, France. multilayer films. A zero magnetostriction permalloy film is deposited on a glass support; then a layer of nonmagnetic metal, usually $\mathrm{Ag}, \mathrm{Au}, \mathrm{Cr}$, or $\mathrm{Pd}$, is evaporated. (The phenomenon has also been observed with $\mathrm{Cu}$ and In.) Finally, a third film of a ferromagnetic metal or alloy with a coercive force higher than that of permalloy, for example, $\mathrm{NiFeCo}$ or pure Co, is evaporated. The films are deposited in a constant magnetic field, and the anisotropy axes of the two ferromagnetic films are usually parallel. We have observed [3]- [5] that, if the intermediate layer has a thickness between 50 and a few hundred angstroms, there is an interaction between the magnetizations of the ferromagnetic layers which we call positive, in the sense that it tends to align the two magnetizations parallel. We have not observed any coupling for a number of insulating intermediate layers $60 \AA$ or more thick [3]. Below this thickness, a coupling which we believe is due to holes in the intermediate is observable. A coupling between $\mathrm{Fe}$ and Fe separated by Pd which appears similar to that reported here has been observed by Trousdale and Lindgren [6]. They observed, by Mössbauer effect, a large exchange field acting on isolated $\mathrm{Fe}^{57}$ atoms a few hundred angstroms from the iron substrate.

\section{Measurement Techniques}

The existence of the coupling is manifested experimentally by the presence of an effective coupling field $H_{i}$ which is related to the superficial energy of interaction $E_{i}$ by

$$
E_{i}=-M H_{i} t \cos \left(\theta_{1}-\theta_{2}\right) .
$$


In (1), $t$ is the thickness of one of the magnetic films, $M$ is its magnetization, and $\theta_{1}$ and $\theta_{2}$ are the magnetization directions of the two films. $E_{i}$ may attain a value of 0.15 $\mathrm{erg} / \mathrm{cm}^{2}$ for intermediate thicknesses near $50 \AA$ and is independent of $t$ for magnetic film thickness in the range we have investigated, that is, the range greater than $350 \AA$.

All the techniques which we have used to measure the coupling are based upon the correctness of (1), which has been confirmed in all cases so far. We have developed five different measurement techniques in our laboratory. The first, measurement of hysteresis loop displacement, has been discussed previously [3], [5]. It and the following three methods are applicable if the coupling and coercive fields satisfy the relation

$$
H_{i}+H_{i}{ }^{\prime}<H_{c}{ }^{\prime}-H_{c}
$$

where $H_{i}$ is the coupling field acting on film $1, H_{i}{ }^{\prime}$ acting on film $2, H_{c}{ }^{\prime}$ is the coercive force of (the hard) film 2 , and $H_{c}$ is the coercive force of film 1 . If (2) is not satisfied, and if the two easy axes are parallel, the effective coercive forces of the two films are the same. If (2) is satisfied, the hysteresis loop of the soft film will be displaced by $H_{i}$ in one sense or the other, so long as the hard film remains saturated. This is shown in Fig. 1. The effective $H_{c}$ of the soft film will be increased by $H_{i}$ if the hard and soft film are switched together.

The second method involves pulse switching and has been employed on films with the structure $\mathrm{NiFe}-\mathrm{Pd}-\mathrm{Co}$, where the $H_{K}$, the anisotropy field, of the hard film is very much greater than that of the soft film. The sample is subjected to the simultaneous action of a continuous field parallel to the easy axis and in the direction opposite to that of the magnetization of the Co film, and of a monopolar pulse field in the hard direction. The pulse field is large enough to switch the NiFe but does not appreciably disturb the Co film. If the continuous field is very small, the magnetization of the NiFe returns after the pulse to the original direction, owing to the coupling. If the field is increased continuously, the read signal becomes zero at a

(a)

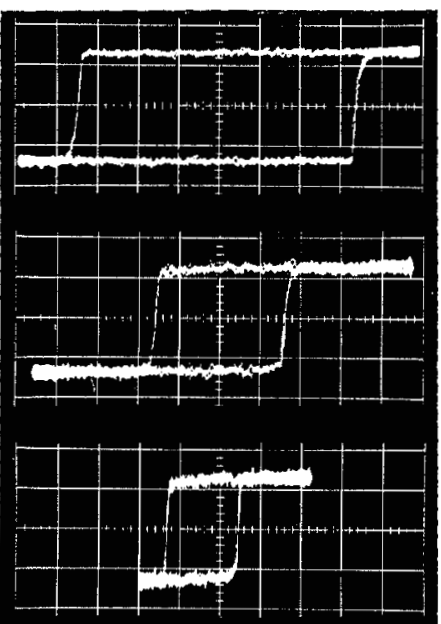

Fig. 1. Hysteresis loops of coupled films, $1 \mathrm{Oe} /$ div. (a) NiFeCo. (b) NiFe, showing the increase in $H_{c}$ when the NiFeCo is switched. (c) NiFe, showing the loop shift. critical value, and then changes polarity as the effect of the field becomes greater than that of the coupling. This behavior is shown in Fig. 2. The critical value of the field is equal to the effective coupling field.

To understand the third and fourth methods, it is necessary to write the total energy of the two films per unit volume of each film.

$$
\begin{aligned}
e & =K_{1} \sin ^{2} \theta_{1}+K_{2} \sin ^{2} \theta_{2}-M H_{x}\left(\cos \theta_{1}+\cos \theta_{2}\right) \\
& -M H_{y}\left(\sin \theta_{1}+\sin \theta_{2}\right)-M H_{i} \cos \left(\theta_{1}-\theta_{2}\right)
\end{aligned}
$$

In writing (3) we have assumed that $M_{1}=M_{2} ; V_{1}=V_{2}$. The two magnetizations and the two volumes are equal. $K_{1}$ and $K_{2}$ are the anisotropy constants of the two films. The first term represents the anisotropy energy of the soft film, the second represents that of the hard film, the last represents the coupling energy, and the others represent the energy of the two magnetic films in the applied field. The stable states are found by setting

$$
\frac{\partial e}{\partial \theta_{1}}=0 ; \quad \frac{\partial e}{\partial \theta_{2}}=0:
$$

$H_{K 1} \sin \theta_{1} \cos \theta_{1}+H_{x} \sin \theta_{1}-H_{y} \cos \theta_{1}$

$$
+H_{i} \sin \left(\theta_{1}-\theta_{2}\right)=0
$$

$H_{K^{2}} \sin \theta_{2} \cos \theta_{2}+H_{x} \sin \theta_{2}-H_{y} \cos \theta_{2}$

$$
-H_{i} \sin \left(\theta_{1}-\theta_{2}\right)=0 \text {. }
$$

We then choose the solutions of (4) which yield positive second derivatives,

$$
\frac{\partial^{2} e}{\partial \theta_{1}^{2}} \text { and } \frac{\partial^{2} e}{\partial \theta_{2}^{2}}
$$

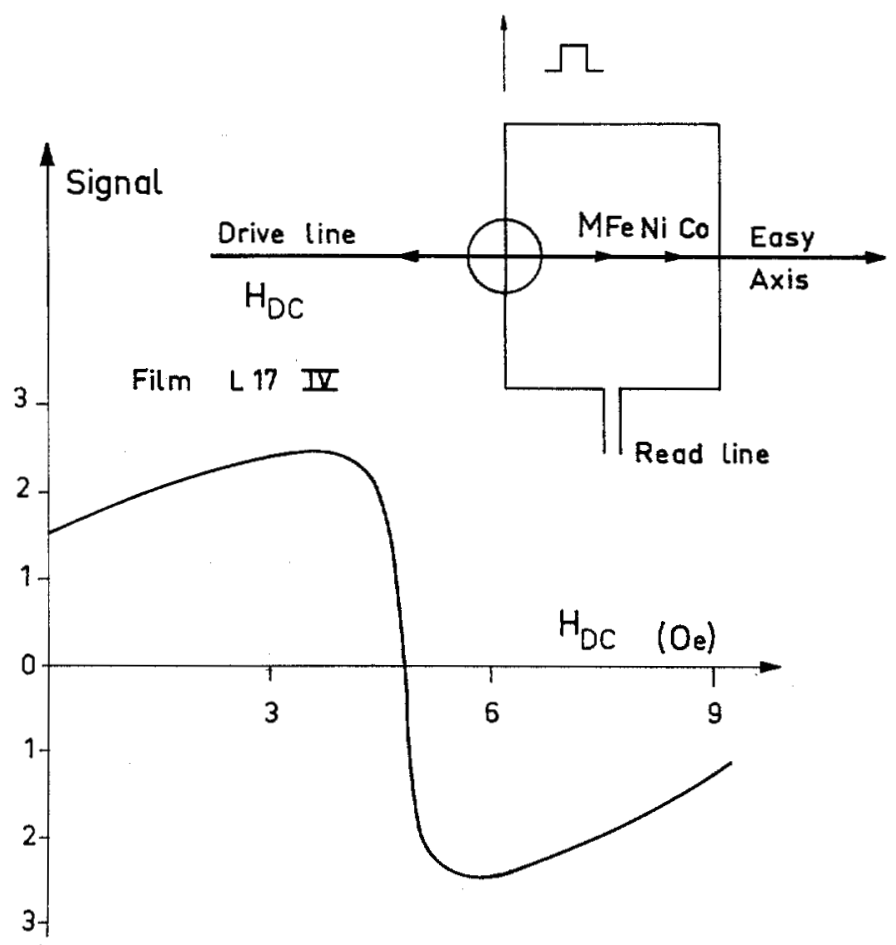

Fig. 2. Experimental arrangement for pulse measurement of coupling, and a typical experimental curve. The coupling field here is 4.8 Oe. 
The third method of measuring the interaction field consists of measuring the hard direction susceptibility in a small applied field. If the coupling field is smaller than $H_{c}$ for the NiFe, it is possible to measure two different susceptibilities when the magnetizations of the two films are parallel and when they are antiparallel. If $\theta_{1}$ is small, and if we can consider $\theta_{2}$ to be equal to zero or $\pi$, (4a) becomes

$$
\begin{gathered}
H_{K} \theta_{1}+-H_{y} \pm H_{i} \theta_{1}=0 \\
\theta_{1}=\frac{H_{y}}{H_{K} \pm H_{i}} .
\end{gathered}
$$

The susceptibility is different for the two cases, with an extrapolation to saturation at $H_{K 1} \pm H_{i}$. The two initial susceptibility curves are shown in Fig. 3(a).

The fourth method which has been applied to multilayers of $\mathrm{NiFe}$ and of $\mathrm{Co}$, where the second film is very much harder than the first, consists of a torque measurement at a field sufficient to turn the magnetization of the $\mathrm{NiFe}$ but insufficient to disturb the magnetization of the hard film. In this case (4a) becomes

$$
\begin{aligned}
H_{K} \sin \theta_{1} \cos \theta_{1}+H \cos \phi \sin \theta- & H \sin \phi \cos \theta_{1} \\
& +H_{i} \sin \theta_{1}=0
\end{aligned}
$$

where $H$ is the applied field and $\phi$ is the angle it makes with the easy direction. The torque per unit volume is given by

$\Gamma=-\frac{\partial e}{\partial \phi}=-H M \sin \phi\left(1+\cos \theta_{1}\right)+H M \cos \phi \sin \theta_{1}$.

If we combine (5) and (6) and assume that $\theta_{1}=\phi$, we find

$$
\Gamma=-H_{K} M \sin \phi \cos \phi-H M \sin \phi-H_{i} M \sin \phi .
$$

$\Gamma$ consists of a uniaxial torque due to the NiFe, a unidirectional torque due to the $\mathrm{Co}$, and a unidirectional torque due to the coupling. If we measure the first two terms on uncoupled films, we can determine the coupling.

These four methods are applicable if the films have different coercive forces, and if (2) is satisfied. When this
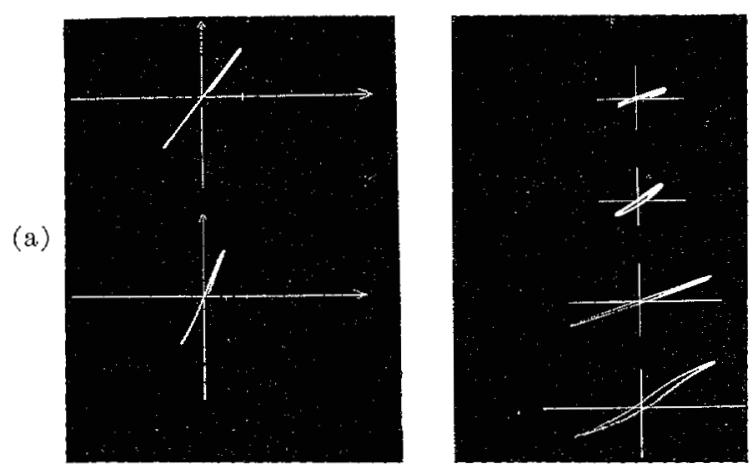

(b)

Fig. 3. Transverse hysteresis loops of coupled film $Y$-21. Characteristics: $H_{c 1}=2.2 \mathrm{Oe}, H_{c 2}=5.6 \mathrm{Oe}, H_{i}=0.4 \mathrm{Oe}, H_{K 1}=$ $7 \mathrm{Oe}, H_{K 2}=21 \mathrm{Oe}$. (a) Low field susceptibility, upper: magnetizations parallel; lower: magnetizations antiparallel. (b) High field loops. was not so, the coupling has been measured by production of the films with their easy directions perpendicular and by measurement of the steady states of the magnetization [7]. If one measures the easy directions of the ensemble, as well as those of each film separately, one may obtain the ratio of the two anisotropies and the ratio of the coupling energy to one of the anisotropies. If the two films have the same anisotropy, this is relatively easy. If the two films do not have the same anisotropy, there is a maximum measurable coupling, A similar technique which could also be used is to measure the biaxial torque as a function of field, as has been done by Siegle for direct exchangecoupled films [8].

Insofar as the five different techniques have been used to measure the positive coupling, the results are in agreement. In certain films having an intermediate layer thickness so great that no positive coupling exists, we have observed a negative loop displacement betwen 0.1 and 10 e.

Bitter and Kerr's studies indicate that this negative loop displacement is due to a difference between the nucleation process for reverse domains when the two magnetizations are parallel and antiparallel. When the magnetizations are parallel, a strong demagnetization field exists, and reverse domains are produced. When they are antiparallel, a stronger field is necessary to nucleate these domains, and thus a loop shift is produced. Since this is an edge effect, it is much stronger than would be predicted by an ellipsoid formula which yields an $H_{d}$ of the order of 2 by $10^{-2}$ Oe. For films with this sort of negative interaction, the susceptibility difference in the hard direction, which provides the third technique for measuring the coupling, does not exist, as only the edges are subject to the coupling.

\section{Expertmental Results and the Origin OF THE COUPLING}

The majority of the studies in our laboratory have been performed with intermediate layers of $\mathrm{Au}, \mathrm{Ag}$, $\mathrm{Cr}$, and $\mathrm{Pd}$. For $\mathrm{Cr}$ and $\mathrm{Pd}$, the coupling energy has been found to be a function of the evaporation temperature, and to increase with increasing evaporation temperature. For $\mathrm{Au}$ and $\mathrm{Ag}$, we have performed some experiments in which we have varied the evaporation parameters. For $\mathrm{Au}$, there is no significant variation of the coupling for evaporations between $30^{\circ} \mathrm{C}$ and a temperature of the order of $260^{\circ} \mathrm{C}$. Above a temperature between $260^{\circ} \mathrm{C}$ and $300^{\circ} \mathrm{C}$, depending on the evaporation technique, the coupling begins to increase. For $\mathrm{Ag}$, it appears that the coupling depends strongly upon the structure, and we are in the process of re-examining this case.

We have observed two types of variation of coupling energy with the intermediate layer thickness. For films of NiFe-metal-NiFeCo the coupling energy decreases linearly with thickness. For NiFe-metal-Co the decrease is nonlinear. The coupling vanishes near $120 \AA$ for $\mathrm{Au}$ and $\mathrm{Cr}$ intermediates and vanishes near $300 \AA$ for $\mathrm{Pd}$ [2], [3], [9].

For all films we have investigated which have intermediate layers of $\mathrm{Cr}$, of $\mathrm{Ag}$, or of $\mathrm{Au}$, the coupling is essentially constant between liquid nitrogen temperature and $350^{\circ} \mathrm{C}$. 
For Pd the coupling decreases with increasing temperature [3], [9].

The unique temperature behavior of the films with $\mathrm{Pd}$ intermediates and the fact that dilute alloys of ferromagnetic metals in $\mathrm{Pd}$ are ferromagnetic suggested that such alloys are produced by diffusion in this case. We have found that this is true. The diffusion is practically negligible at room temperature, but is already detectable at $100^{\circ} \mathrm{C}$. By comparing the properties of these films with those of films with PdCo intermediate layers, we have found that all the coupling in this case can be attributed to the ferromagnetic alloy produced by diffusion [10]. On this basis, we can expect that there will be no bulk diffusion into the $\mathrm{Au}$ or the $\mathrm{Ag}$ at room temperature.

For films with $\mathrm{Au}$ and $\mathrm{Cr}$ intermediates, we have dissolved away the magnetic material and examined the films in the electron microscope [9]. We have found that whenever the films with $\mathrm{Cr}$ intermediates show coupling there are holes along grain boundaries and that these disappear at the same time as the coupling. For the Au films, there is no evidence for holes in a large number of films which show coupling. This does not preclude the possible existence of very small holes of less than $15 \AA$ diameter.

On the basis of the experimental results presented here, it seems almost certain that the coupling with $\mathrm{Cr}$ intermediates is due to the passage of magnetic material along the grain boundaries and the formation of bridges between the two films. It is not impossible that this is also true for $\mathrm{Au}$, but the relative independence of the coupling of evaporation temperature, and the electron microscopic evidence, do not tend to confirm this hypothesis. The coupling here may be due to an indirect interaction, produced by the polarization of the conduction electrons in the intermediate layer. A possible mechanism of this interaction has been suggested by Dreyfus, et al. [11].

\section{Switching Properties}

In discussing the techniques for measuring the coupling, we have already treated a certain number of switching properties of the coupled films. We have done this by considering limiting or approximate cases, in order to solve (4a) and (4b). However, in order to calculate in general the stable states and low-frequency switching of coupled films, it is necessary to resort to a computer. The technique we have used is somewhat different from that used by Chang [12]. With $K_{1}, K_{2}, H_{i}, H_{x}$ and $H_{3}$ fixed one chooses a value for $\theta_{1}$. The condition $\partial e / \partial \theta_{2}=0$ is easily resolved. Knowing $\theta_{2}$ we calculate $\partial e / \partial \theta_{1}$, and look for the condition $\partial e / \partial \theta_{1}=0$. This yields $\theta_{1}$ and $\theta_{2}$, the stable states. With the stable states, we have calculated the critical curve and a number of hysteresis loops.

For single films, the critical curve has two functions [13]. First, in passing from the interior to the exterior, one stable state disappears each time a part of the curve is crossed. Second, the tangents to the curve are the stable magnetization directions. For the coupled films, we take the critical curve as the locus of points where the number of stable states decreases. The tangents to this curve are only approximately the stable directions of magnetization.

Without the performance of any calculations, it is possible to say certain things concerning the relationship between the critical curve of a single film and that of the coupled films. As the coupling is treated as an effective field, the total effective field is the vector sum of the applied field and the coupling field. The resultant critical curve cannot be displaced by a distance greater than $H_{i}$ from the original curve.

The critical curve of a pair of films calculated for $K_{2} / K_{1}$ $=3.6$ and $H_{i} / H_{K}=0.23$ is exhibited in Fig. 4. Going outward from the center, one passes gradually from four stable states to one. In the region indicated by the dotted lines there are two stable states, but the energy varies very slowly as a function of $\theta_{1}$ and $\theta_{2}$. Thus the stable states are difficult to calculate and their stability is weak. This, as we shall see, is reflected in the experimental results.

One half of the critical curve for film $Y-21$ is shown in Fig. 5. The remaining half is the mirror image of what is shown. For this film $K_{2} / K_{1}$ is near 3.6 , but $H_{i} / H_{K}$ is somewhat less than 0.23 . The two interior critical curves are displaced by $2 H_{i}$, where $H_{i}$ is defined from the value along the horizontal axis, in agreement with theory. However, the form of the curve near the horizontal axis is not in agreement with theory, as wall displacement rather than rotation is the dominant reversal mechanism.

Figure 6 shows the critical curves of two other films. Figure 6(a) shows dispersion of the critical field in the region where theory indicates weak stability. We suppose that this is in agreement with the theory, as slight perturbations seriously modify these states. Figure $6(\mathrm{~b})$ shows the irregular form of the critical curve near the horizontal axis. This is not predicted at all by the theory and is presumably due to interactions between domain walls in the two films, as the two show the same effective coercive force.

The predicted hysteresis loops in the hard direction are shown in Fig. 7. The two curves for film 1 extrapolate to $H_{K 1} \pm H_{i}$. Film 2 saturates near $H_{K 2}-H_{i}$ if $H_{i}$ and $H_{K 1}$ are both much smaller than $H_{K 2}$. The experimental curves in the hard direction for film $Y 21$ are shown in Fig. 3. The low field extrapolation agrees with theory. However, the loop turns down and opens up when the two magnetizations are in different quadrants. This indicates a wall displacement, which is not understood at present.

We have also calculated the hysteresis loops with a continuous field in the hard direction and with an alternating field in the easy direction; the component of the magnetization is measured in the hard direction, that is $\sin$ $\theta_{1}$. These curves are shown in Fig. 8. For single films, such loops are symmetrical; here, there are two asymmetries: the crossing point is displaced to $-H_{i}$, and one maximum is more pronounced than the other. The curve becomes reversible at slightly less than $H_{K 1}$.

The experimental results, shown in Fig. 9, are in agreement with the theory, as regards the general form and the displacement of the loops. The principal maximum resembles the theoretical one, the secondary maximum does not. This can be explained in the same way as the small 


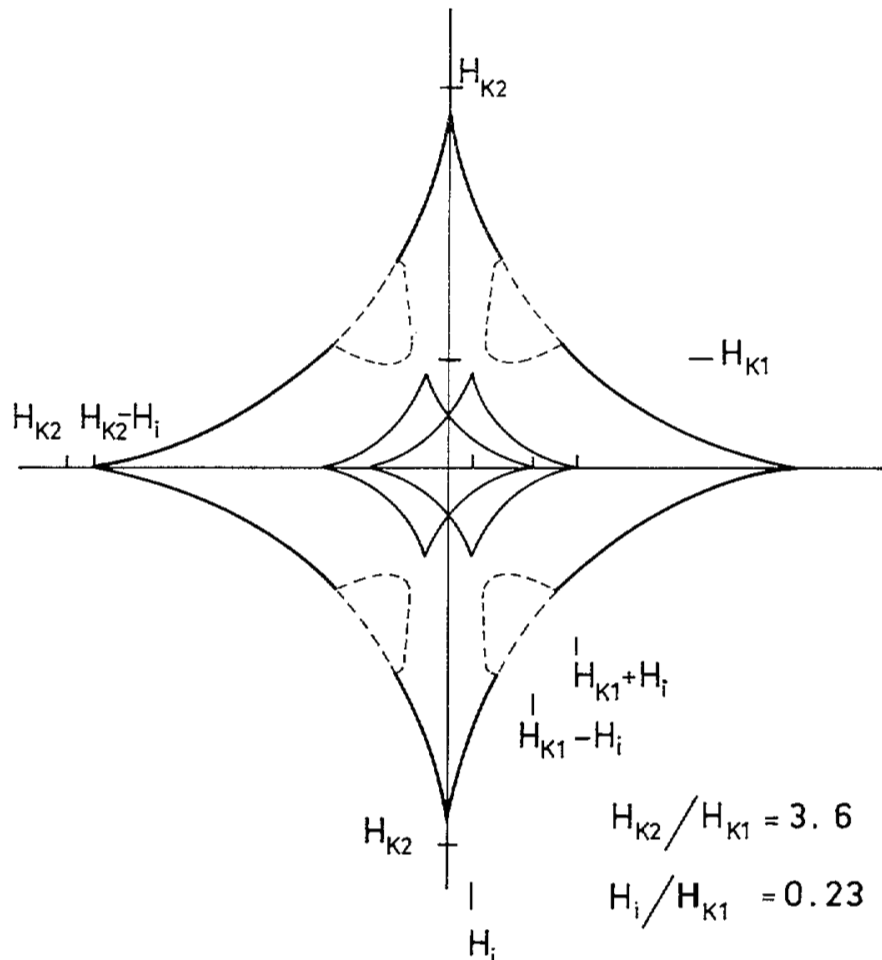

Fig. 4. Theoretical critical curve for a coupled film pair, with $H_{K 2} / H_{K 1}=3.6, H_{1} / H_{K 1}=0.23$. (a)

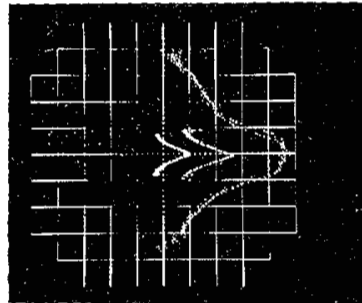

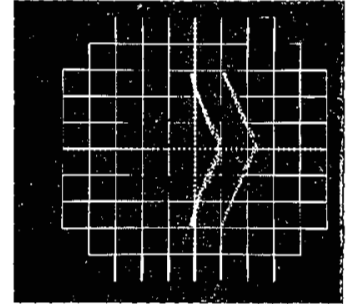

(b)

Fig. 5. One half of the critical curve for film $Y$-21. (a) Interior and exterior curves. (b) Interior curves only.
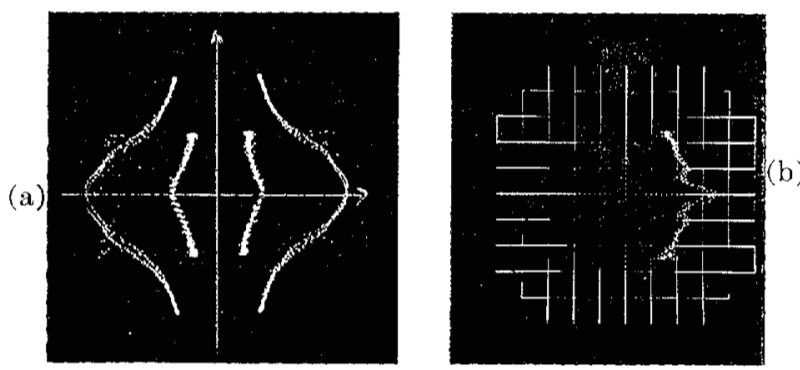

Fig. 6. Critical curves for two films. (a) $Y-11, H_{c 1}=13 \mathrm{Oe}$ $H_{\mathrm{c} 2}=11 \mathrm{Oe}, K_{K 1}=7 \mathrm{Oe}, H_{K 2}=24 \mathrm{Oe}, H_{i}=1.6 \mathrm{Oe}$ (b) $Y-18$ $H_{c 1 e f f}=2 \mathrm{Oe}, H_{c 2 e f f}=2 \mathrm{Oe}, H_{K 1}=7.5 \mathrm{Oe}, H_{K 2}=18 \mathrm{Oe}$.

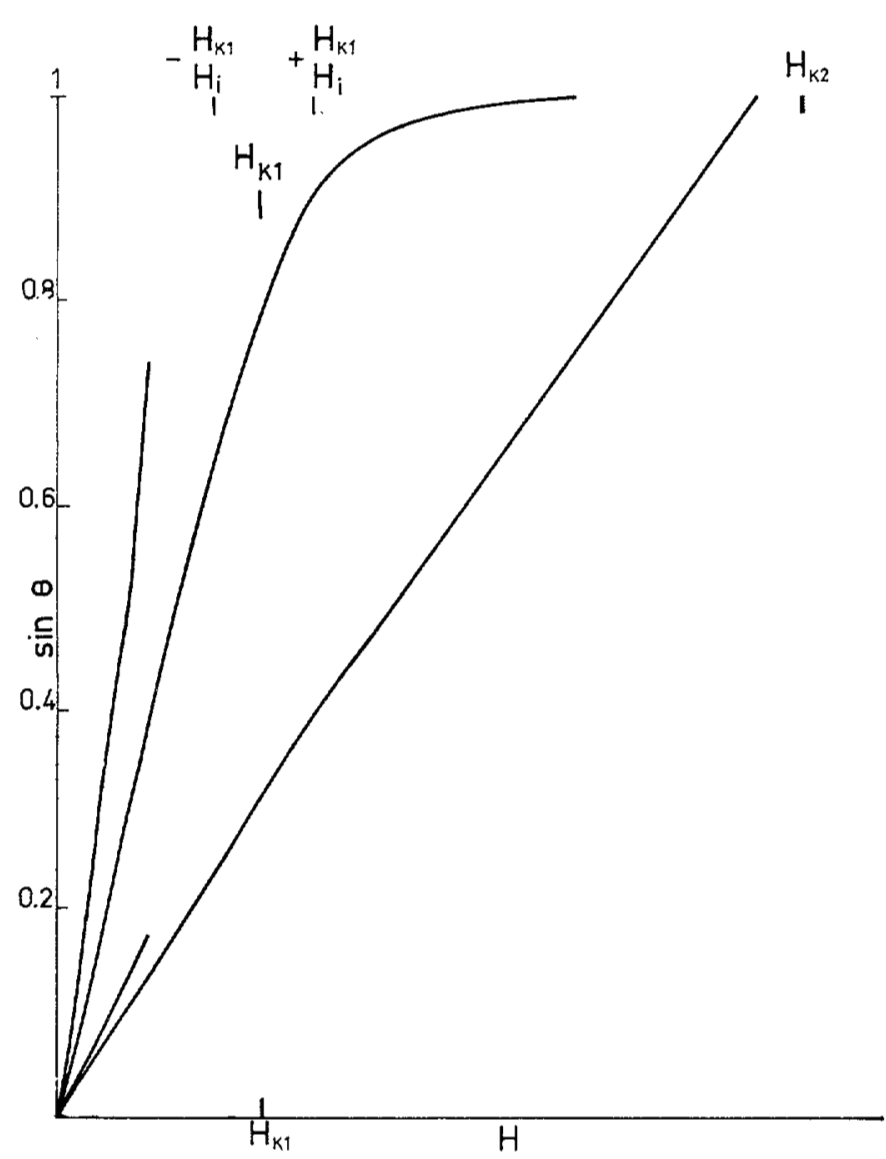

Fig. 7. Theoretical transverse (hard direction) hysteresis loops, for the same conditions as the critical curve of Fig. 4 .

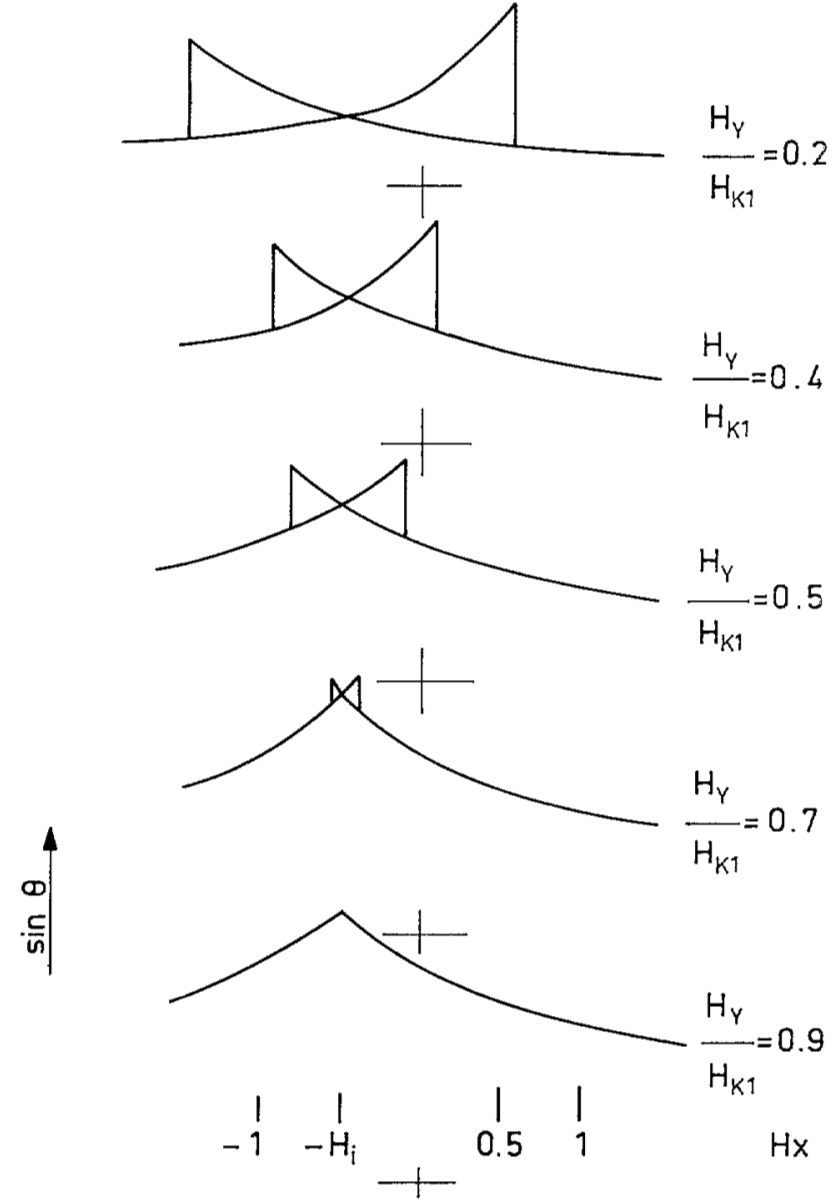

Fig. 8. Theoretical hysteresis loops with de field and sensing in the hard direction, and ac field in the easy direction. Same conditions as Figs. 4 and 7 . 

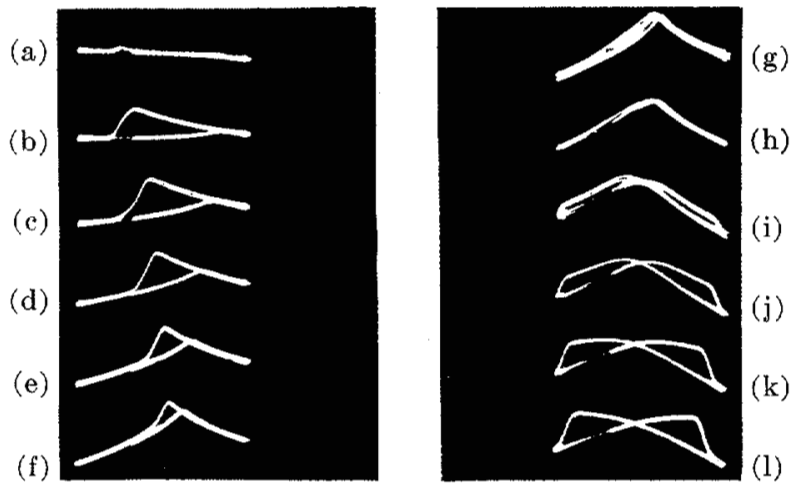

Fig. 9. Experimental loops for film $Y$-21 for varying values of the de field. (a) $H_{y}=0.15$ Oe. (b) $H_{y}=0.7 \mathrm{Oe}$ (c) $H_{y}=1.8 \mathrm{Oe}$. (d) $H_{y}=3.0 \mathrm{Oe}$. (e) $H_{y}=4.0 \mathrm{Oe}$ (f) $H_{y}=5.3 \mathrm{Oe}$. (g) $H_{y}=$ 7.7 Oe. (h) $H_{y}=8.7$ Oe. (i) $H_{y}=110 \mathrm{Oe}$ (j) $H_{y}=15.5 \mathrm{Oe}$ (k) $H_{y}=22.5$ Oe. (l) $H_{y}=27 \mathrm{Oe}$

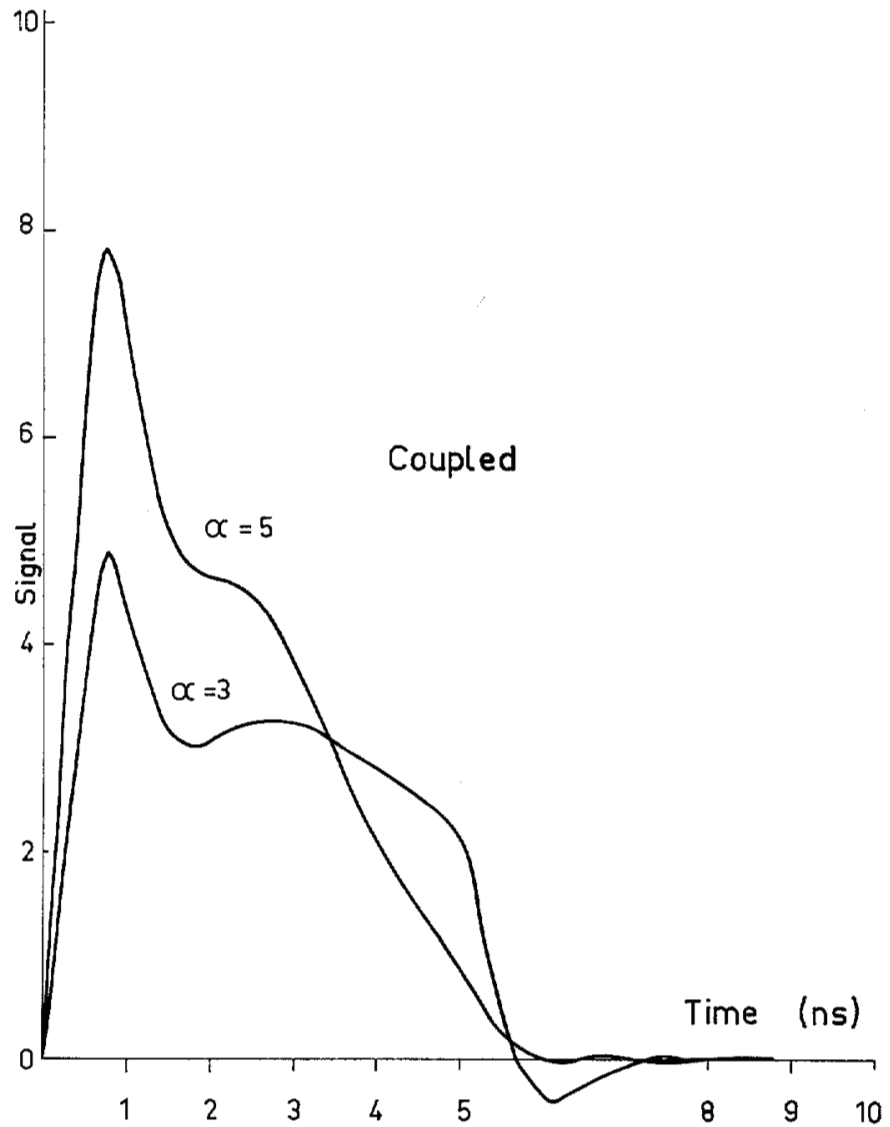

(a) (a)

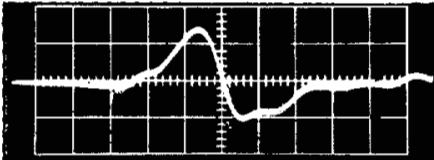

(b)

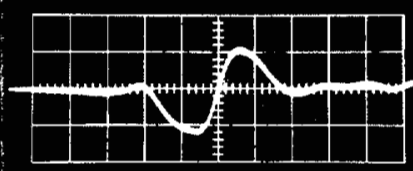

(c)

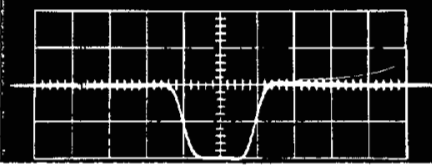

Fig. 10. Operation of a coupled film as a bit in NDRO, $10 \mathrm{~ns} / \mathrm{div}$ repetition rate 2 Mc. (a) " 1 ". (b) " 0 ". (c) Word pulse.

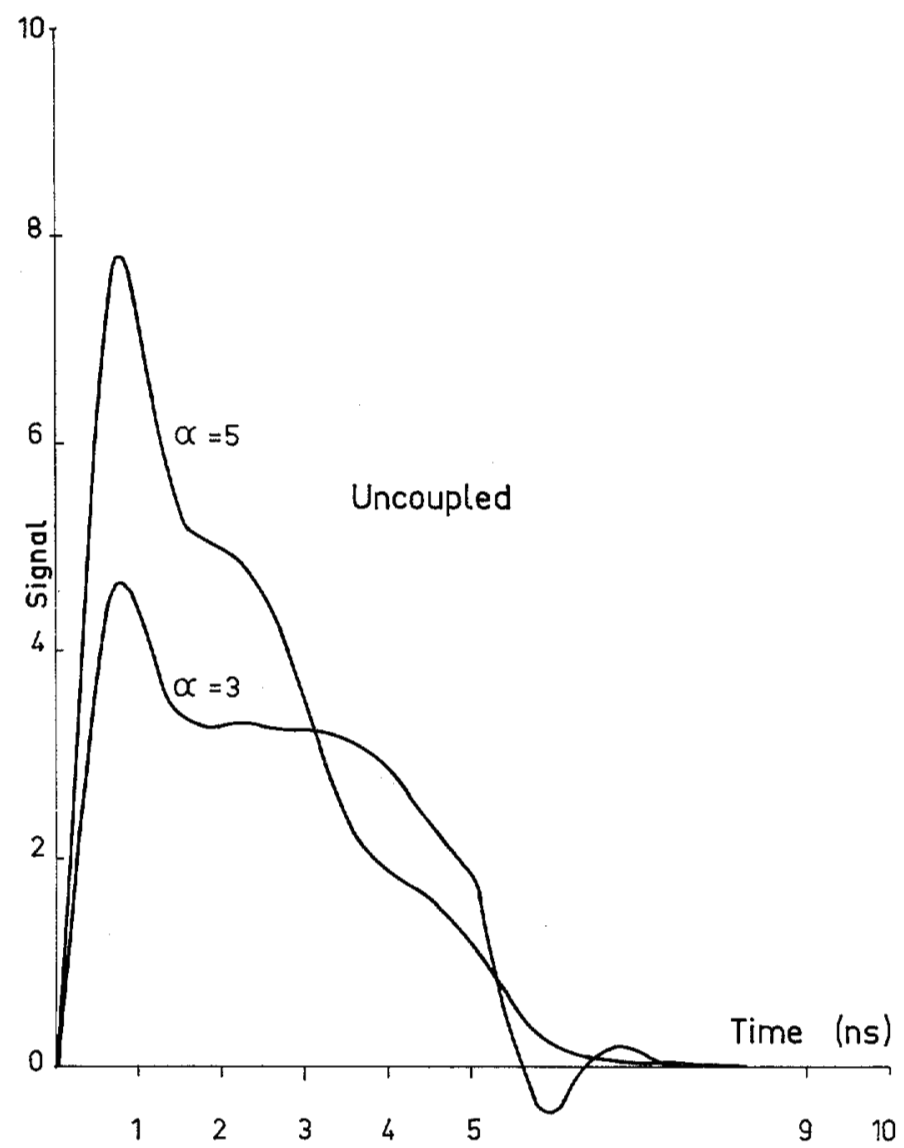

(b)

Fig. 11. Theoretical switching signals for film pairs, with $H_{K_{1}}=2.5 \mathrm{Oe}, H_{K 2}=5 \mathrm{Oe}, \lambda=1.5 \times 10^{8}$ seconds ${ }^{-1}$, showing the total signal of the two films, word pulse rise time, 5 ns. $\alpha$ is the maximum value of the field in Oe. (a) Coupled. (b) Uncoupled.

negative coupling discussed earlier. Reverse domains are nucleated when the magnetizations are parallel and are not nucleated when the magnetizations are in different quadrants. But this type of model cannot explain the wall effects observed in the hard direction.

We have previously proposed that these coupled films may be used as nondestructive memory elements [5]. The read operation is performed by applying a pulse field in the hard direction large enough to switch the soft film, but not the hard film. The soft film then returns, owing to the coupling. We have confirmed that the information can be read nondestructively, and in Fig. 10 we show the signal obtained at a $2-\mathrm{Mc}$ repetition rate.

Calculations based on the Landau-Lifschitz equation [14] have been performed for a coupled and for an uncoupled pair of $\mathrm{NiFe}$ and $\mathrm{NiFeCo}$ films. The same value of $\lambda$ was assumed for both. The switching curves for two values of the applied field, assuming 5 ns rise times, 
are shown in Fig. 11. The rise time and signal strength are almost the same in both the coupled and the uncoupled. cases, but the critical damping of the coupled films takes place near $H_{K^{2}}$. Experimentally, the rise times and signals observed for the coupled films are in reasonable agreement with theory.

Feldtkeller [15] has recently pointed out that the simultaneous existence of the indirect positive coupling and a coupling between domain walls due to demagnetizing fields can greatly modify the coercive force and creeping threshold. In particular, for two $250-\AA \mathrm{NiFe}$ films, separated by $\mathrm{Au}$, he found that the coercive force and the creeping threshold were both increased. We have also found that for $\mathrm{NiFe}$ and $\mathrm{NiFeCo}$ with nearly the same effective $H_{c}$, separated by Au, the creep threshold for fields applied at reasonably large angles to the easy axis is increased. In contrast, we find that the coercive force is decreased by the coupling, as has been found by Middelhoek [16] for NiFe films separated by SiO.

Further, the optimum thickness conditions for creeping in the coupled films are not at all the same as those for single films. For example, we have chosen $1800 \AA$ as the thickness of the nickel iron and attempted to vary the thickness of the NiFeCo to minimize creeping. We find that a thickness of NiFeCo near $400 \AA$, for which we observe Néel walls, is not as favorable for preventing creeping as a thickness of $900 \AA$. This problem is still being investigated.

\section{ACKNOWLEDGMENT}

The authors wish to thank J. Devenyi for performing the electron microscope investigations, and Dr. N. Gastinel and A. Eberhard of the Institut des Mathématiques Appliquées of Grenoble for programming and performing the machine calculations. We also wish to thank Drs. $\mathrm{H}$ Juretschke and D. O. Smith for helpful discussions and suggestions.

\section{REFERENCES}

[1] C. Maurain, J. Phys., vol. 1, p. 90, 1902.

[2] - ibid., p. 151

[3] J. C. Bruyère, et al., "Sur un nouveau phénomène de couplage entre couches minces ferromagnétiques séparées par un matériau non ferromagnétique," Acad. Sci. (France), vol. 258, pp. 841-844, January 1964.

[4] --, "Interactions magnétiques entre deux couches minces ferromagnétiques séparées par une coche de chrome ou de palladium d'épaisseur inférieure à $300 \AA$," Acad. Sci. (France), pp. 1423-1425, February 1964.

[5] "A coupling phenomenon between the magnetization of two ferromagnetic thin films separated by a thin metallic film-application to magnetic memories," IEEE Trans. on Magnetics, vol. MAG-1, pp. 10-12, March 1965.

[6] W. L. Trousdale and R. A. Lindgren, "Range of ferromagnetic exchange interaction in non magnetic films on iron substractes," J. A ppl. Phys., vol. 36, pp. 968-970, March 1965.

[7] A. Yelon, et al., "Coupling between two uniaxial magnetic films with perpendicular easy directions, separated by a nonferromagnetic layer," Proceedings of the International Conference on Magnetism. London: Inst. of Physics and the Physical Soc., 1964, pp. 791-793.

[8] W. T. Siegle, "Exchange coupling of uniaxial magnetic thin films," J. A ppl. Phys., vol. 36, pp. 1116-1117, March 1965.

[9] J. C. Bruyère, et al., "Coupling effect between the magnetizations of two thin layers separated by a thin non metallic layer," J. Appl. Phys, vol. 36, pp. 944-945, March 1965.

[10] O. Massenet, to be published in Compt. Rend. Acad. Sci. (Paris).

[11] B. Dreyfus, et al., "Long-range magnetic coupling in metals;" Phys. Rev. Letters, vol. 13, pp. 342a-343a, September 1964.

[12] H. Chang, "Analysis of static and quasidynamic behavior of magnetostatically coupled thin magnetic film," IBM $J$. Res. and Dev., vol. 6, pp. 419-429, October 1962.

[13] E. W. Pugh and T. O. Mohr, "Properties of ferromagnetic films," in Thin Films. H. G. F. Wilsdorf, Ed. Metals Park, Ohio: Am. Soc. Metals, 1964, pp. 195-226.

[14] D. O. Smith, "Static and dynamic behavior of permalloy films," J. A ppl. Phys., vol. 29, pp. 264-273, March 1958.

[15] E. Feldtkeller, "Wand bewegunssfeld stärken in magnetischen mehrfachschichten," Z. Angew. Phys., vol. 18, pp. 532-534, $196 \overline{0}$.

[16] S. Middelhoek, "Properties of $\mathrm{Ni}-\mathrm{Fe}$ double films," to be published.

\title{
Comparison of Magnetic Behavior of Cylindrical and Flat Films from Kerr Effect Probe Measurements
}

\author{
D. B. DOVE, T. R. LONG, ANd J. E. SCHWENKER, MeMber, IEeE
}

\begin{abstract}
The magnetic characteristics of flat and cylindrical permalloy films are compared. $1000 \AA$-flat films and $10000-\AA$ cylindrical films electrodeposited onto 5-mil-diameter wires show very similar magnetic properties. Geometrical differences arise largely in the relative ease or difficulty with which fields may be applied to the films and signals extracted. The greater thickness of the cylindrical films described gives rise to enhanced magnetostatic effects apparent in the Kerr effect probe measurements and to strong self-shielding against nonaxial external fields.
\end{abstract}

Manuscript received March 22, 1965. This paper was presented at the 1965 INTERMAG Conference, Washington, D. C.

The authors are with Bell Telephone Labs., Inc., Murray Hill,
TANY STUDIES of the magnetic behavior of flat 11 permalloy films have been reported previously [1]. In contrast, there have been relatively little data reported pertaining to cylindrical permalloy films [2]-[6]. It is the object of this report to discuss the application of a highresolution Kerr effect probe to the measurement of permalloy films electroplated on a copper-coated 5 -mil-diameter beryllium-copper wire and to compare the observed measurements and effects with those obtained from similar experiments on flat, evaporated permalloy films. 